\title{
Expected Tracking Performance with the HL-LHC ATLAS Detector
}

\author{
Kira Abeling* on behalf of the ATLAS Collaboration \\ II. Physikalisches Institut, Georg-August-Universität Göttingen, Germany \\ E-mail: kira.abelingecern.ch
}

The High-Luminosity LHC (HL-LHC) aims to increase the LHC data-set by an order of magnitude in order to increase its potential for discoveries and precision measurements. Starting approximately in 2026, the HL-LHC is expected to reach the peak luminosity of $7.5 \times 10^{34} \mathrm{~cm}^{-2} \mathrm{~s}^{-1}$ which corresponds to conditions where up to 200 inelastic proton-proton collisions can occur per bunch crossing which is approximately five times the current number of collisions per bunch crossing. To cope with the large radiation doses and high pileup, the current ATLAS Inner Detector will be replaced with a new all-silicon Inner Tracker which will cover up to $|\eta|=4$. These proceedings summarise recent results regarding the expected tracking performance of the Inner Tracker. The impact of tracking on reconstruction of selected physics objects is shown.

European Physical Society Conference on High Energy Physics - EPS-HEP2019 -

10-17 July, 2019

Ghent, Belgium

\footnotetext{
${ }^{*}$ Speaker.
} 


\section{Introduction}

With the High-Luminosity LHC (HL-LHC) data-taking starting in 2026, a significant increase in luminosity is planned. This offers new possibilities to observe rare physics processes and to perform searches for new physics but also gives new challenges for the detectors. The ATLAS detector [1] will therefore be upgraded accordingly. After the first upgrade of the Inner Detector (ID) in 2014 with an additional layer, the Insertable B-Layer (IBL) [2], the ID will be replaced in 2025 with the Inner Tracker (ITk) [3,4] to cope with the requirements for the HL-LHC, namely the high data rate and radiation dose.

\section{ITk layout}

The layout for the ITk has been first presented in the Technical Design Reports in Refs. [3,4] but have been updated since then to include new insights and recent developments [5]. In Figure 1a, the current layout of the planned ITk is shown. It is a silicon-only detector with a pseudorapidity coverage of $|\eta| \leq 4.0$, split up into a pixel (inner part) and strip detector (outer part). The total size is limited by the free space inside the ATLAS calorimeters. An important development is the reduction of the pixel pitch size to $50 \times 50 \mu \mathrm{m}^{2}$ or $25 \times 100 \mu \mathrm{m}^{2}$, compared to the IBL which had $50 \times 250 \mu \mathrm{m}^{2}$ pixel pitches. The ID and the ITk are compared in Table 1. For a more detailed description of the layout, see Refs. [3-5].

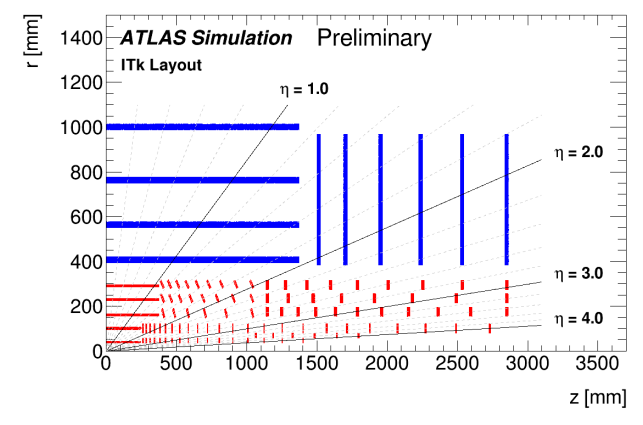

(a) Schematic layout of the ITk

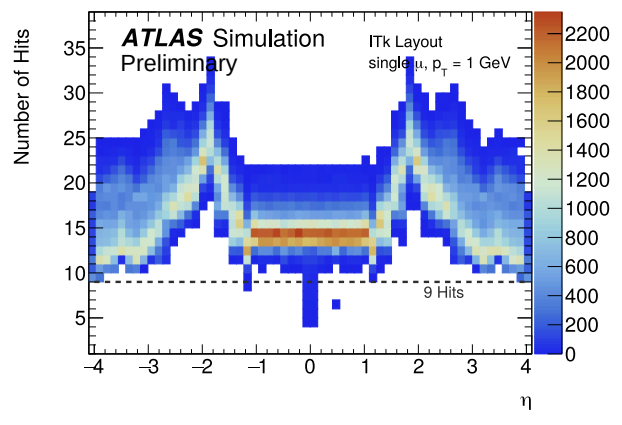

(b) Number of silicon hits

Figure 1: (a) ITk layout in the $R-z$ plane, where red shows the pixel and blue the strip detector, and (b) the expected number of silicon hits as a function of $\eta$ for $p_{\mathrm{T}}=1 \mathrm{GeV}$ single muon tracks [5].

This layout is designed to reach at least nine silicon hits across the whole $\eta$ range. As shown in Figure $1 \mathrm{~b}$, this is the case for nearly all tracks with $p_{\mathrm{T}}=1 \mathrm{GeV}$. The small fraction of tracks in the central region with less than nine hits can be explained by gaps between pixel and strip modules.

\section{Track Reconstruction}

ITk tracks are reconstructed following the procedure described in Ref. [4]. Analogue clustering determines the cluster position for pixels to improve the resolution by considering the charge deposited in the individual pixels. During reconstruction, tracks have to pass certain quality criteria which are summarised in Table 2 for three different $\eta$ regions in the ITk and the ID in Run-2. 


\begin{tabular}{ccc}
\hline & Inner Detector & Inner Tracker \\
\hline subdetectors & pixel, strip, TRT & pixel, strip \\
coverage & $|\eta| \leq 2.5$ & $|\eta| \leq 4.0$ \\
$R_{\min }$ & $33 \mathrm{~mm}$ & $39 \mathrm{~mm}$ \\
$R_{\max }^{\text {silicon }}$ & $512 \mathrm{~mm}$ & $1000 \mathrm{~mm}$ \\
pixel pitch & $50 \times 250 \mu \mathrm{m}^{2}(\mathrm{IBL})$ & $50 \times 50 \mu \mathrm{m}^{2}$ or \\
& $50 \times 400 \mu \mathrm{m}^{2}(\mathrm{else})$ & $25 \times 100 \mu \mathrm{m}^{2}$ \\
\hline
\end{tabular}

Table 1: Comparison of basic quantities between the current ID and the ITk [1-5].

\begin{tabular}{ccccc}
\hline \multirow{2}{*}{ Requirement } & \multicolumn{3}{c}{ Pseudorapidity interval } & \multirow{2}{*}{ Run-2 } \\
& $|\eta|<2.0$ & $2.0<|\eta|<2.6$ & $2.6<|\eta|<4.0$ & \\
\hline pixel+strip hits & $\geq 9$ & $\geq 8$ & $\geq 7$ & $\geq 7$ \\
holes & $\leq 1$ & $\leq 1$ & $\leq 1$ & $\leq 2$ \\
$p_{\mathrm{T}}[\mathrm{MeV}]$ & $>900$ & $>400$ & $>400$ & $>400$ \\
$\left|d_{0}\right|$ & $<2 \mathrm{~mm}$ & $<2 \mathrm{~mm}$ & $<10 \mathrm{~mm}$ & $<10 \mathrm{~mm}$ \\
$\left|z_{0}\right|$ & $<20 \mathrm{~cm}$ & $<20 \mathrm{~cm}$ & $<20 \mathrm{~cm}$ & $<25 \mathrm{~cm}$ \\
\hline
\end{tabular}

Table 2: Summary of the track requirements for ITk and Run-2 used in the presented studies [5].

\section{Tracking Performance}

In Figure 2a, the expected tracking efficiency for the ITk and the current ID are shown using simulated $t \bar{t}$ events. The tracking performance for $|\eta| \leq 1.5$ is comparable for the ITk and ID, while the ITk outperforms the ID significantly for $|\eta|>1.5$. Considering the fake rate of tracks, i.e. fraction of tracks that cannot be matched to a stable simulated particle, Figure $2 \mathrm{~b}$ shows clearly that the ITk outperforms the ID by roughly one order of magnitude due to high number of silicon precision hits [5]. Note that for both distributions the different run conditions are already taken into account by using a different average number of interactions per bunch crossing with $\langle\mu\rangle=200$ (HL-LHC conditions) for the ITk and $\langle\mu\rangle=20$ (Run-2 conditions) for the ID.

Figure 3 shows the resolution of two important track quantities: the transverse impact parameter, $d_{0}$ and the longitudinal impact parameter, $z_{0}$, for low and high energetic muon tracks and the two pixel pitch sizes under consideration. For low-energy muons ( $p_{\mathrm{T}}=1 \mathrm{GeV}$, top row), there is no visible difference between the different pixel pitch sizes since the resolution is dominated by material effects [5]. Compared to the ID, the $d_{0}$ resolution is worse which relates directly to the $6 \mathrm{~mm}$ larger inner radius of the ITk (see Table 1). Note that smaller radii are still under investigation. For $z_{0}$, the performance is very similar. For high-energy muons $\left(p_{\mathrm{T}}=100 \mathrm{GeV}\right.$, bottom row), the ITk generally outperforms the ID. Differences in the resolution for the pixel pitch size are of the order of a factor 2 and the pitch size associated to the best performance depends on the impact parameter under investigation.

Tracking information is used in many algorithms to determine other quantities. In Figure 4a, the hard-scatter vertex reconstruction efficiency is shown. For the ITk vertex reconstruction, the 


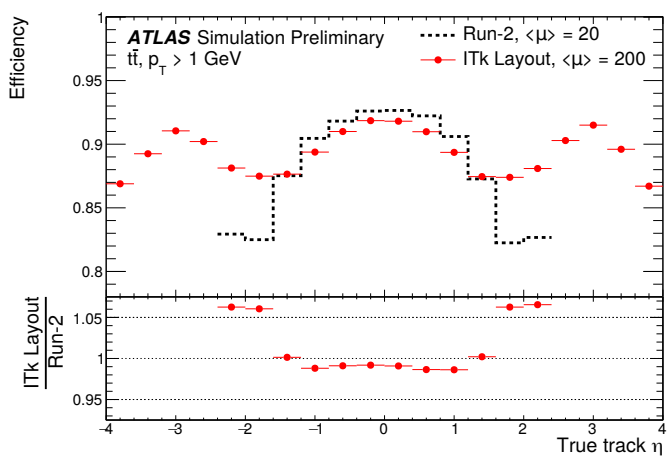

(a)

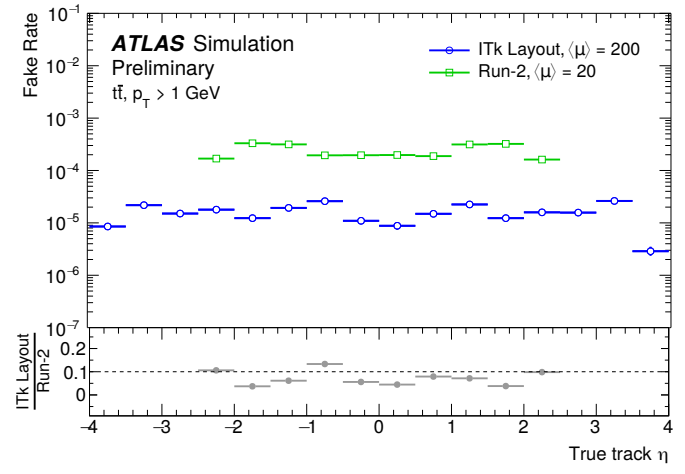

(b)

Figure 2: (a) Tracking efficiency and (b) fake rate as a function of the true track $\eta$ [5].
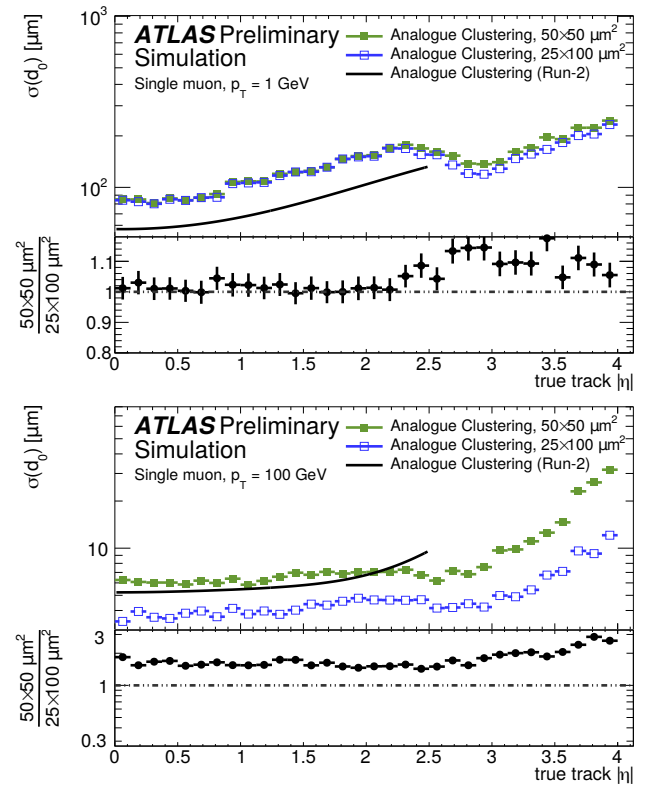

(a) Transverse impact parameter
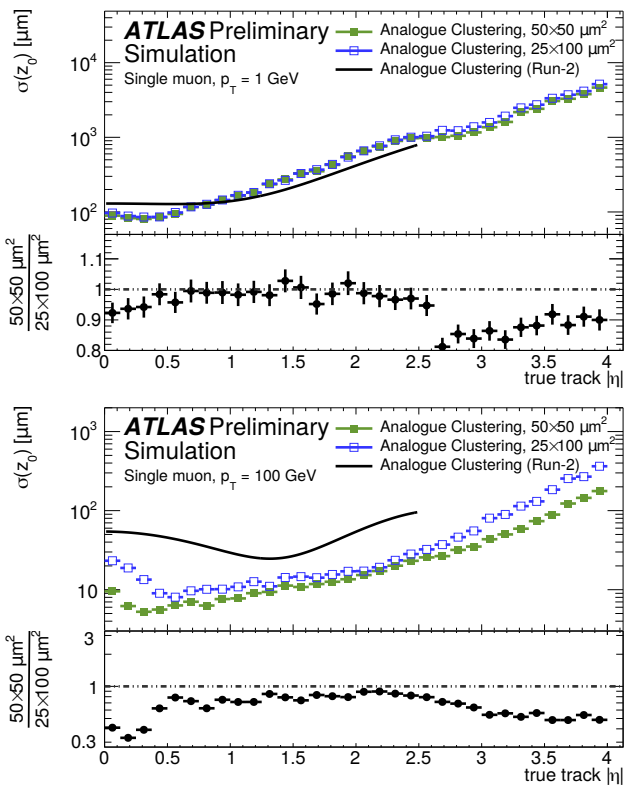

(b) Longitudinal impact parameter

Figure 3: Resolution of the most important track impact parameters as a function of the true track $\eta$ evaluated using muons with $p_{\mathrm{T}}=1 \mathrm{GeV}$ (top) and with $p_{\mathrm{T}}=100 \mathrm{GeV}$ (bottom) [5].

Adaptive Multi-Vertex Finder [6] is used, which is very similar to the Run-2 algorithm [7] but fits several vertices simultaneously. The efficiency in the ITk is expected to stay at a constant high level ( $\geq 99 \%$ ), while the efficiency decreases rapidly for the ID when increasing the pile-up density. The improved vertexing will be helpful in the discrimination between hard-scatter tracks and pile-up tracks. The choice of the ITk pixel pitch size only has a minor impact.

For the $b$-tagging [8,9] in Figure 4b, only the $25 \times 100 \mu \mathrm{m}^{2}$ pixel pitch is shown since a better performance than for the $50 \times 50 \mu \mathrm{m}^{2}$ pixel pitch is expected due to the improved $d_{0}$ resolution. Despite the larger inner radius, the ITk can improve the $b$-tagging performance of the ID and, additionally, allows the possibility for forward $b$-tagging. 


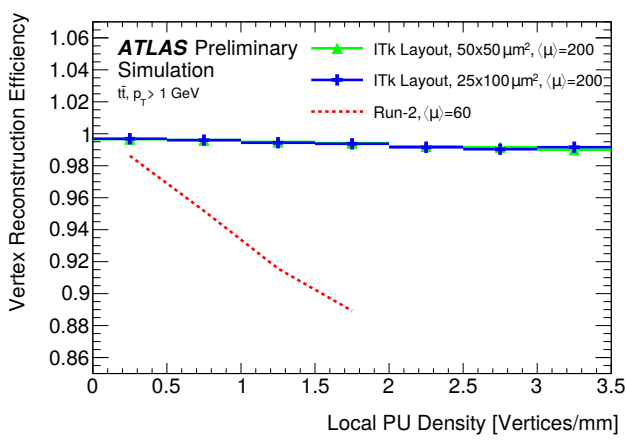

(a) Vertex reconstruction efficiency in $t \bar{t}$ events depending on the pile-up density for both pixel pitch sizes of the ITk and the current ID.

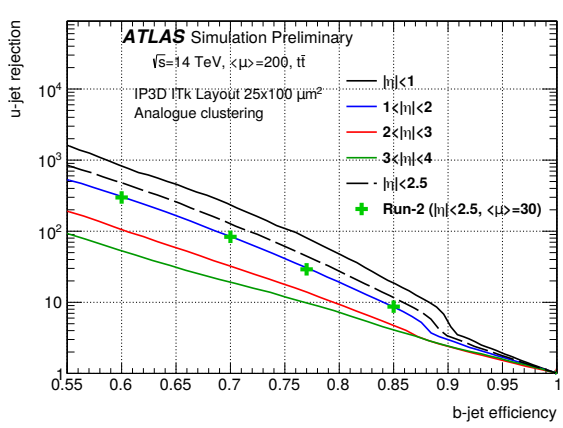

(b) $b$-tagging efficiency versus light jet rejection for the $25 \times 100 \mu \mathrm{m}^{2}$ pixel pitch of the ITk in three different $\eta$ regions and the ID in Run-2.

Figure 4: Two example use cases for tracking information [5].

\section{Conclusions}

The studies presented here show the expected tracking performance of the ATLAS detector at the HL-LHC, where the current ID will be completely replaced by the ITk. Compared to the tracking in Run-2, the ITk is expected to perform the same or even better despite the more challenging run conditions. In particular, the extended pseudorapity range from $|\eta| \leq 2.5$ to $|\eta| \leq 4.0$ offers a new window for analyses.

\section{References}

[1] ATLAS Collaboration, The ATLAS Experiment at the CERN Large Hadron Collider, JINST 3 (2008).

[2] ATLAS Collaboration, ATLAS Insertable B-Layer Technical Design Report, CERN-LHCC-2010-013 (2010), cds.cern.ch/record/1291633.

[3] ATLAS Collaboration, Technical Design Report for the ATLAS Inner Tracker Strip Detector CERN-LHCC-2017-005 (2017), cds. cern.ch/record/2257755.

[4] ATLAS Collaboration, Technical Design Report for the ATLAS Inner Tracker Pixel Detector, CERN-LHCC-2017-021 (2017), cds . cern. ch/record/2285585.

[5] ATLAS Collaboration, Expected Tracking Performance of the ATLAS Inner Tracker at the HL-LHC, ATL-PHYS-PUB-2019-014 (2019), cds . cern. ch/record/ 2669540.

[6] E. Bouhova-Thacker et al. (ATLAS Collaboration), Vertex reconstruction in the ATLAS experiment at the LHC, in proceedings of 2008 IEEE Nuclear Science Symposium, Medical Imaging Conference and 16th International Workshop on Room-Temperature Semiconductor X-Ray and Gamma-Ray Detectors (2008), cds.cern.ch/record/1139840.

[7] ATLAS Collaboration, Reconstruction of primary vertices at the ATLAS experiment in Run 1 proton-proton collisions at the LHC, Eur. Phys. J. C77 (2017), hep-ex:1611.10235.

[8] ATLAS Collaboration, Performance of b-jet identification in the ATLAS experiment, JINST 11 (2016), hep-ex:1512.01094.

[9] ATLAS Collaboration, Optimisation and performance studies of the ATLAS b-tagging algorithms for the 2017-18 LHC run, ATLAS-PHYS-PUB-2017-013 (2017), cds . cern . ch/record/2273281. 